\title{
QUALITY PERCEPTION OF ISLAMIC HIGHER EDUCATION INSTITUTION SERVICES: \\ Experience of International Students
}

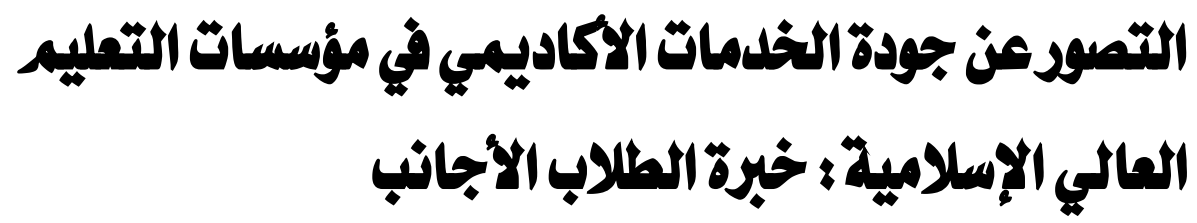

A6d.Haris and $\mathcal{N} u r$ Asnawi

Universitas Islam Negeri Maulana Malik Ibrahim Malang, Indonesia

E-mail: prof.abdharis@yahoo.com

\section{Abstract}

This study aims to formulate a special dimension of service quality in Islamic higher education institutions in shaping Islamic culture according to the perceptions of international Muslim students. Using a quantitative approach with exploratory factor analysis, it involved 100 international Muslim students taken from multi-stage proportionated stratified random sampling (probability sampling). The results showed that based on the experience of international Muslim students, the service quality of Islamic higher education institutions included the dimensions of teaching staff competencies, administrative staff competencies, Islamic environment, physical facilities and information technology-based services, all of which can work together in shaping Islamic culture. To support the vision of the internationalization of Islamic higher education institutions, managers must pay attention to these dimensions as a representation of the quality of service felt by international Muslim students to meet their expectations during their studies at Islamic higher education institutions in Indonesia. 
يهدف هذا البحث إلى صياغة بعد خاص لجودة الخدمات في مؤسسات التعليم العالي بناءً على تصور الطلاب المسلمين الأجانب في بناء الحضارة بعدارة

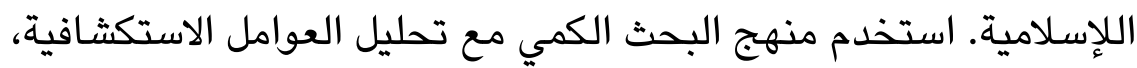

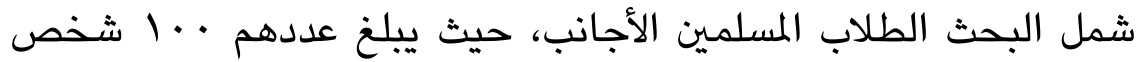
ويتم أخذهم باستخدام عينة عشوائية طبقية (أخذ العينة الاحتمالية). وأظهرت النتائج أن جودة الخدمات في مؤسسات التعليم العالي الإسلامية بناءا على تجرية الطلاب المسلمين الأجانب تشمل بعد كفاءة أعضاء هيئة

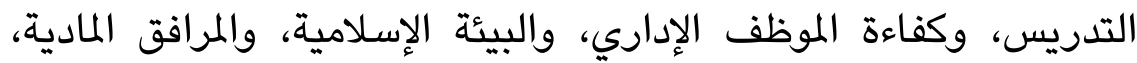

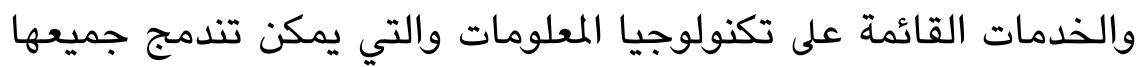

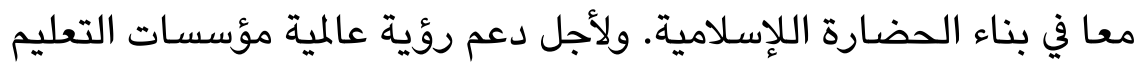

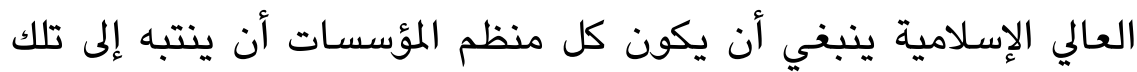

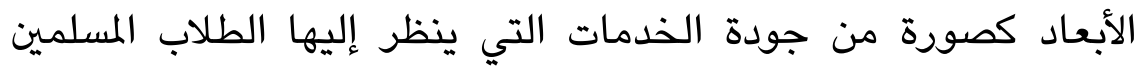
الأجانب عند تحقيق توقعاتهم أثناء الدراسة في مؤسسات التعليم العالي الإسلامية بإندونيسيا.

Keywords: service quality; quality management systems; international students; interculturality

Received: April 11, 2020; Accepted: June 29, 2020

المقدمة

اليوم، أصبح موضوع النقاش حول جودة خدمات التعليم العالي بإندونيسيا قضية مهمة، بسبب عدم وجود أي مؤسسة التعليم العالي بإندونيسيا

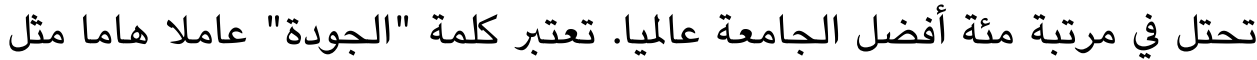

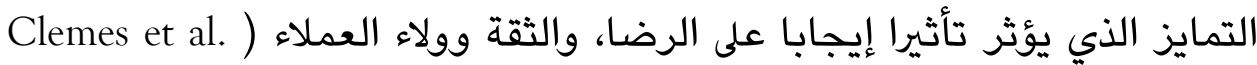
2008; Aga and Safakli 2007; Omar et al. 2009 الخدمات في التعليم العالي الإسلامي روحاً مشتركة لمتطلبات المؤسسة في المنافسة 
الحازمة بين مؤسسات التعليم العالي، متطلبات أصحاب المصلحة، روح العالمية

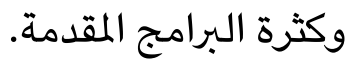

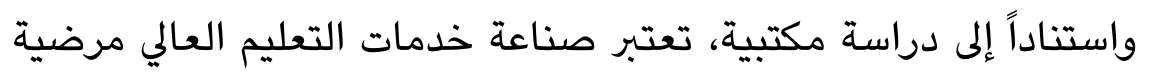

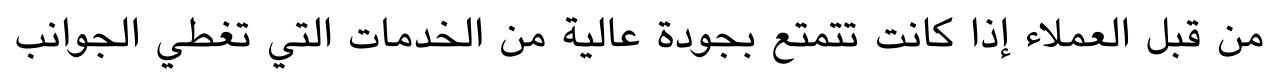
الأكاديمية وغير الأكاديمية ( Arokiasamy and Abdullah 2012; Dado et al. 2012; Asaduzzaman et al. 2013; Saglik et al. 2014; Hanaysha et al. 2011; Chen and Chen 2014; Brown and (Gruber et al. 2010 (Mazzarol 2009; Melewar and Akel 2005; Ivy 2001 .(Holdford and Reinders 2001) ويصفة عامة، فإن المقيم على جودة الخدمات في التعليم العالي هو الطالب بوصفه عميل رئيسي (Sakthivel et al. 2005; Telford dan Masson 2005) ومراقب الجودة الفعال (Hill et al. 2003). وأصبح التحقق من أدوات قياس

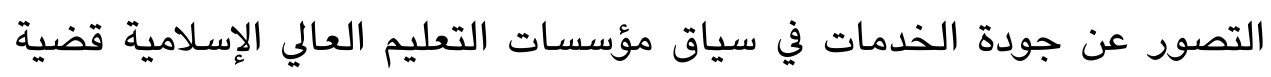

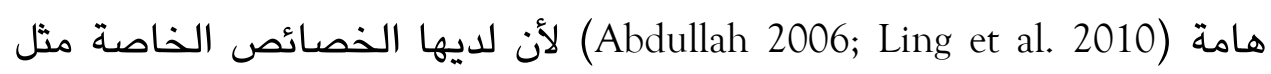

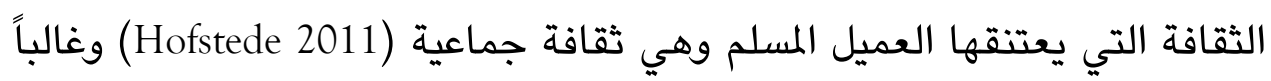

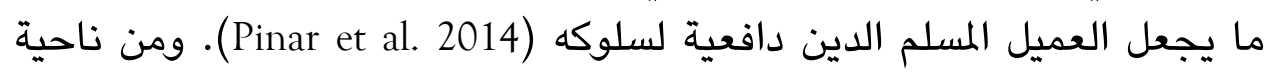
أخرى، كان التحقق من قياس التصور عن جودة الخدمات في مؤسسات التعليم التصني

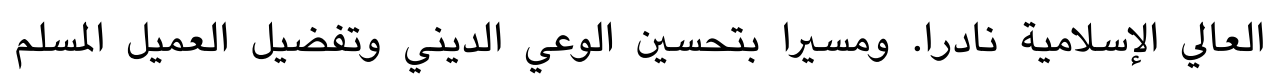

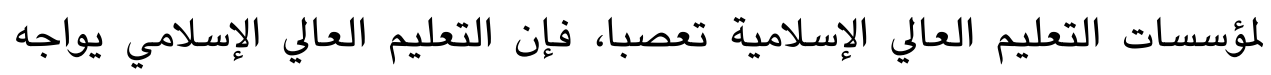
تحديات متزايدة وينبغي أن يكون متكيفا مع متطلبات العمات العملاء.

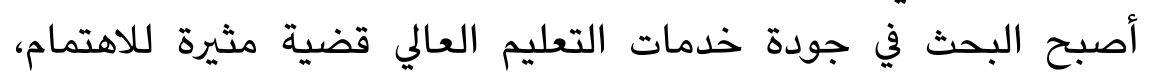

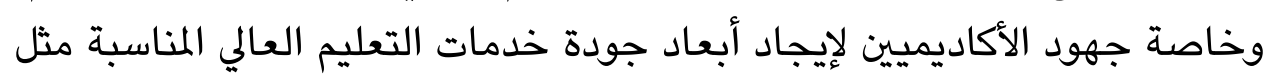

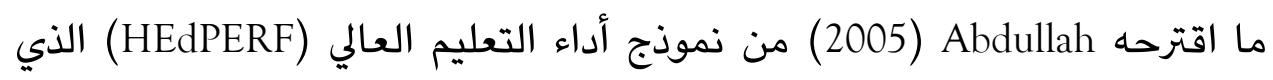
يتكون من الجانب الأكاديمي وغير الأكاديمي والواقعي والعاء العاطفي. نموذج Sultan and Tarafder (Annamdevula and Bellamkonda 2012)HiEdQUAL Phed-) (2007) (اموا بتطوير النموذج القائم على أداء جودة خدمات التعليم العالي 
الذي ادعى أن تكون أداة شاملة بالمقارنة مع نموذج أداء التعليم العالي (model (HEdPERF)

واستناداً إلى الدراسة المكتبية، تبين أن أبعاد الجودة في التعليم العالي لا

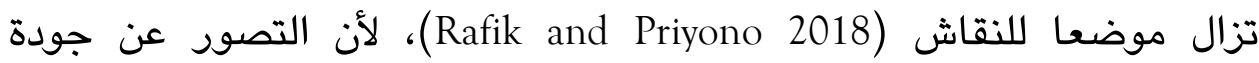

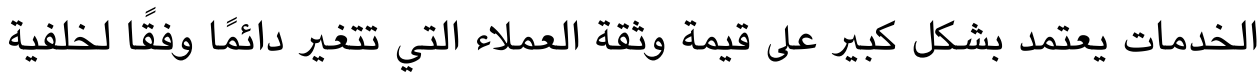

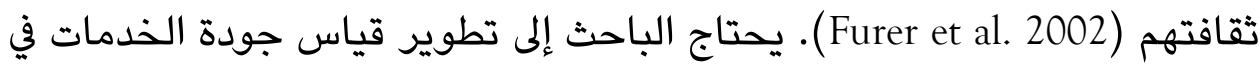

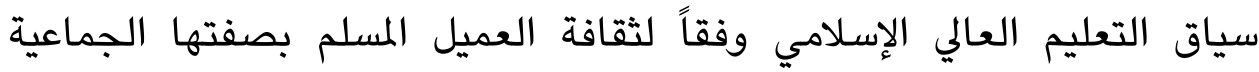

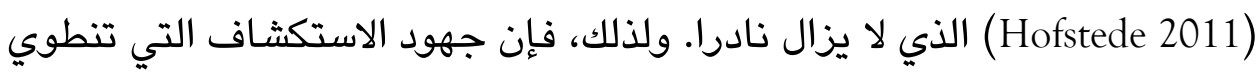

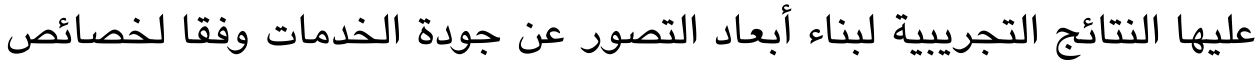

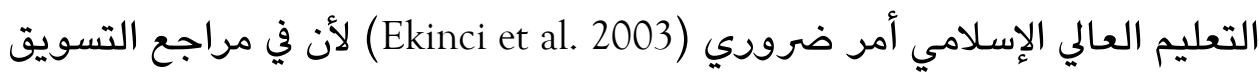
الدين والثقافة هما دافعيان لسلوك العملاء (Pinar et al. 2014).

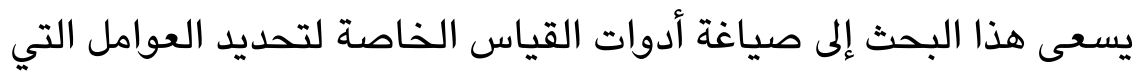

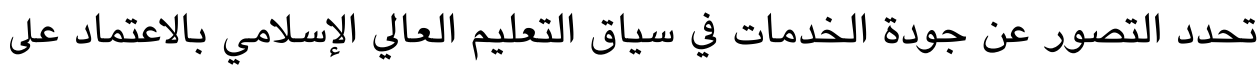

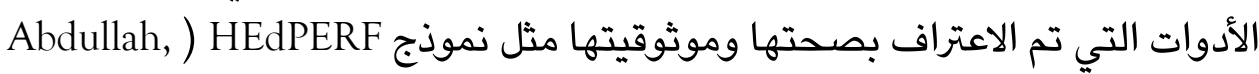
HiEdQUAL ؛(Sultan and Tarafder 2007) Phed-model 2005

.(Annamdevula and Bellamkonda 2012)

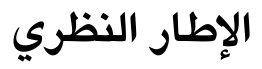

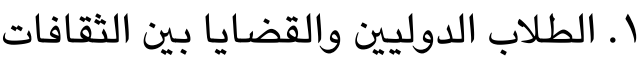

يحتاج الطلاب الدوليون إلى خدمات أكاديمية بخصائص مختلفة، خاصة الفافة

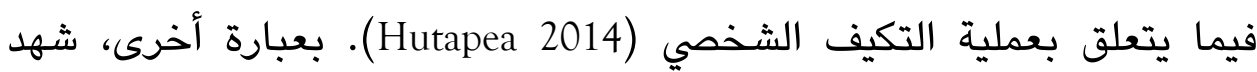

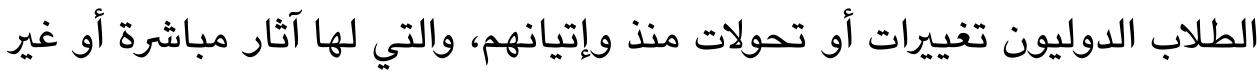
مباشرة على الضغط الذي سماه Redden كصدمة ثقافية (Hutapea 2014).

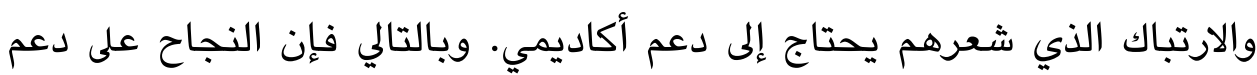

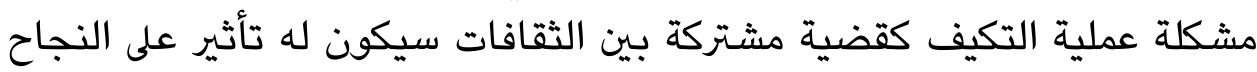

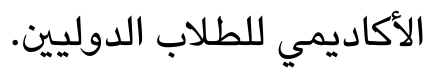


خدمات أكاديمية جيدة ستؤثر تئثيرا مباشرا في تنمية كفاءة التكيف للطلاب الدوليين لدعم الصدمة الثقافية (Mitasari and Istikomayanti 2018). ومع ذلك، التأثير المباشر هو عملية التكيف اللغوي المستخدمة في الأمور المستخدمة في نيل الخدمات الأكاديمية (Pyvis and Chapman 2005).

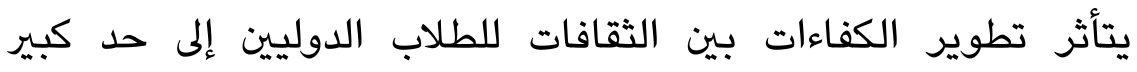
باستدامة عملية التكيف مع القدرة الاستيعابية الكافية للخدمات الأكاديمية (Dunworth et al. 2019)

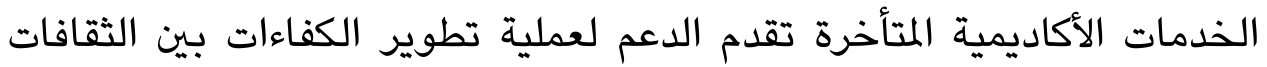
للطلاب الدوليين. قال Firco و Lockhart (2018) أن إحدى الخطوات الخدات لتحات لتحقيق

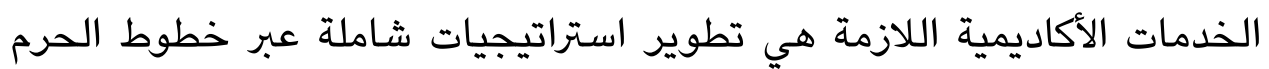

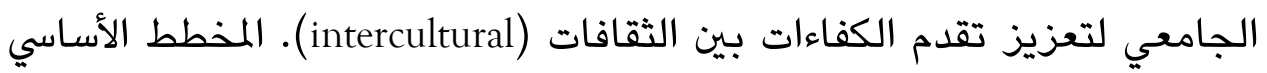

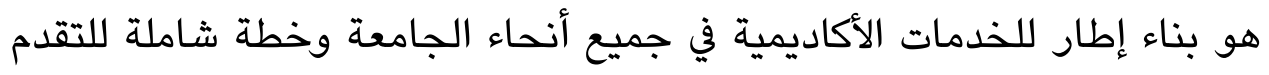

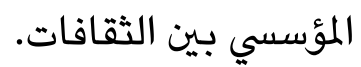

مع مزيد العدد من الطلاب الدوليين في مؤسسات التعليم العالي الإسلامية،

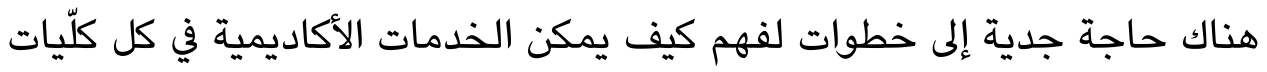

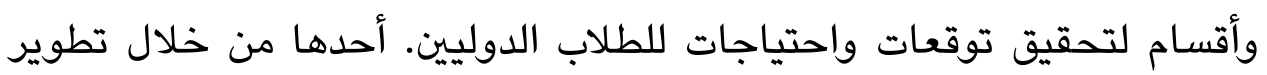

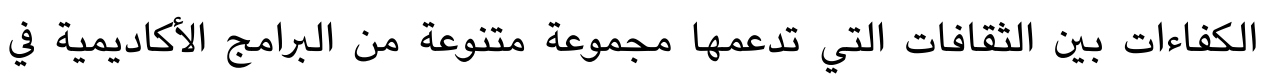

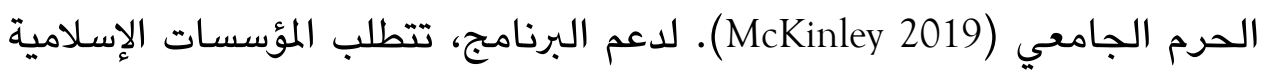

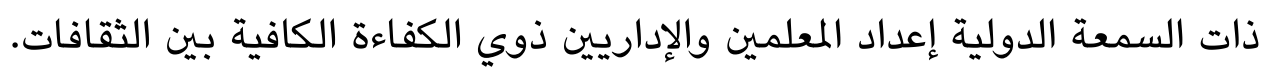

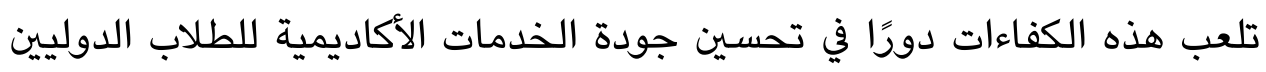
(Zenner and Squire 2020) ب. التعليم العالي الإسلامي

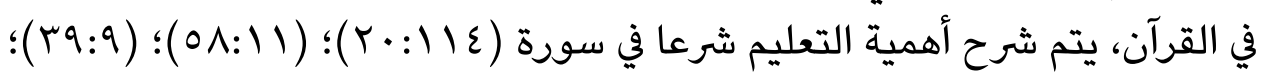

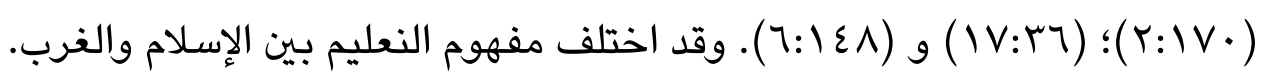

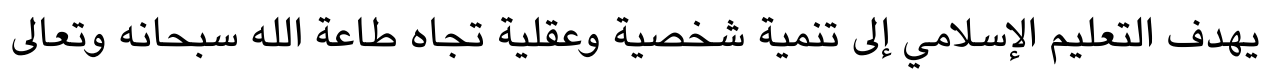
(Alavi 2007)، في حين التعليم الغربي يهدف إلى تعزيز التفكير النقدي المستقل 
والليبرالي (Hussain 2007). تعتمد منهجية العلم في الغرب على قواعد التجربة والعقلانية والمادية وتجاهل الكتب المقدسة، وبالعكس فإن منهجية العلم في

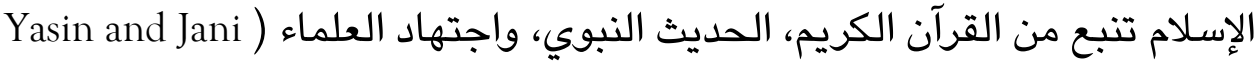

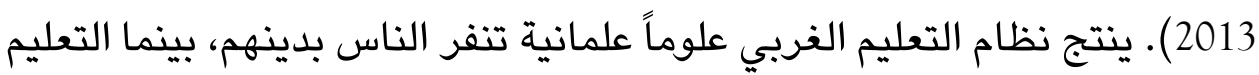
الإسلامي يوجه نحو التوازن بين الجوانب الروحية والجسدية وزيادة الإيمان بالله سبحانه وتعالى (2007 (Hussain). إن وجود التعليم العالي الإسلامي بإندونيسيا له رسالة تطوير المعرفة الحديثة

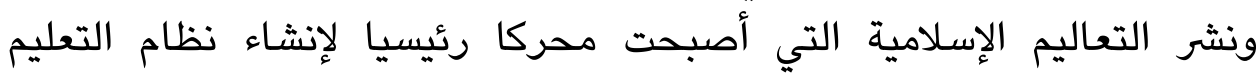

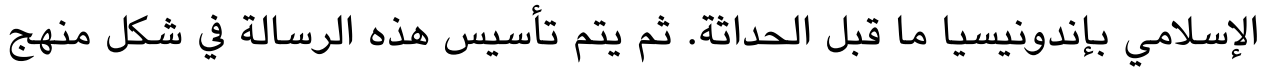

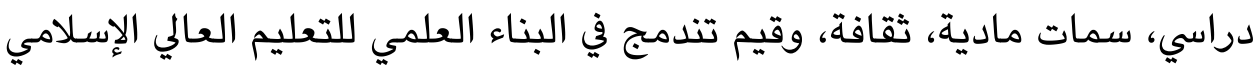

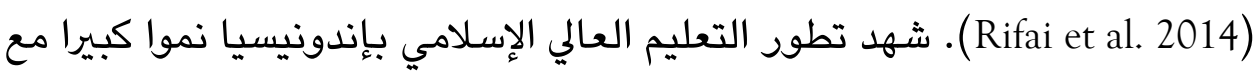

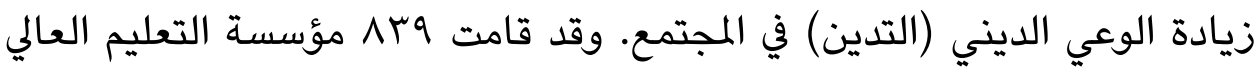

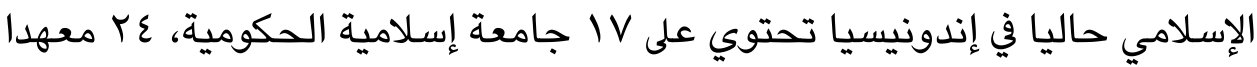

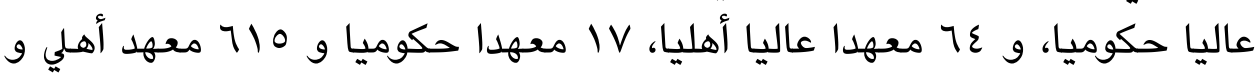

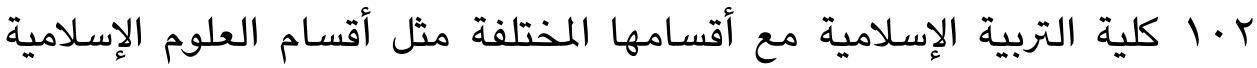

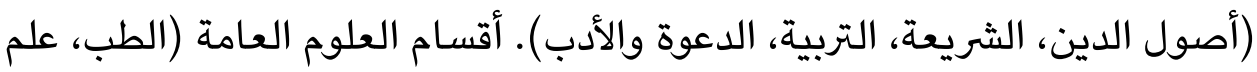
النفس، الاقتصاد، العلوم الإنسانية الاجتماعية، والعلوم الطبيعية) ( Direktorat .(Pendidikan Tinggi Islam-DIKTIS 2019

\section{أبعاد جودة خدمات التعليم العالي}

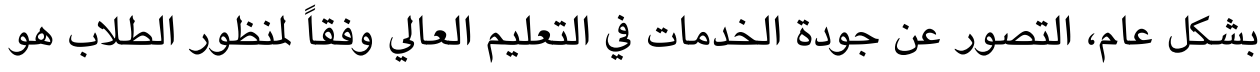
شيء مجرد (Sumaedi et al. 2012) بحيث يحاول الباحثون القياس من خلال نماذج مناسبة مع الظروف الاجتماعية الجغرافية. توجد دراسات تسعى إلى إيجاد أبعاد جودة الخدمات في التعليم العالي. وجد أوليا وأسفين وول (1996) ستة البعائ أبعاد؛ وهي الملموسة، الكفاءة، الموقف، المحتوى، التسليم، والموثوقية. ويحدد رسلي وآخرون (2012) خمسة أبعاد وهي ملموسة وموثوقة واستجابة وتأكية ولكدية 
وتعاطفية. تمكن عبد الله (2005) من العثور على مقياس محدد يسمى HEdPERF

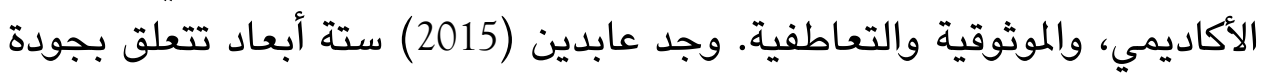
الخدمات في التعليم العالي الإسلامي، وهي: محاضري، والمائ، منهج تعليمي، إدارية، مرافق، مكتبة وبيئة إسلامية. وتشمل أبعاد جودة التعليم العالي وفقا لـ أنامديفولا وبيلاميكوندا (2012) محتوى التدريس والدورة التدريبية، والخدمات الإدارية، وتهمل

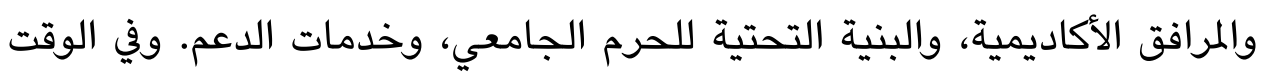

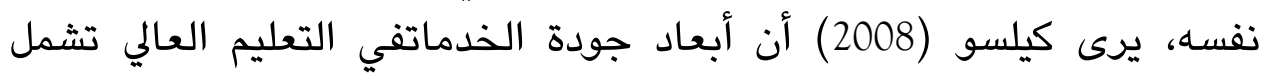

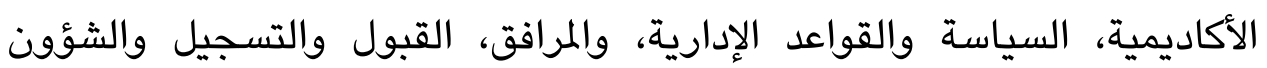

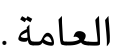
ولا يزال نموذج SERVQUA) (Parasuraman et al. 1985) أساسا للبحث عن e.g Calvo-Porral et al. 2013; Ezeokoli ) جودة الخدمات المتعلقة بالتعليم العالي and Ayodele 2014 ). وحتى الآن، تم اقتراح مدخل متعدد الأبعاد لقياس جودة

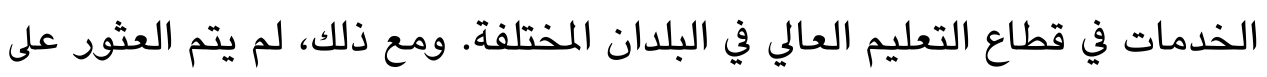

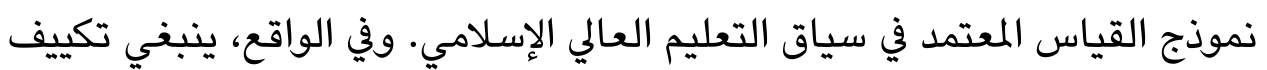

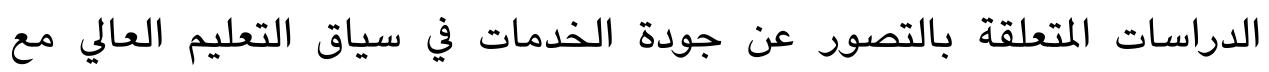

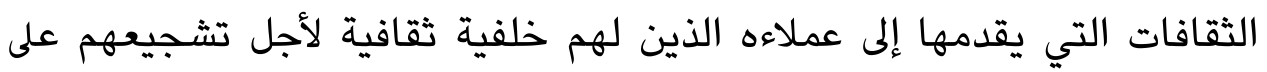
التقييم.

\section{منهجية البحث}

وقد صاغ هذا البحث الأبعاد الخاصة لجودة خدمات التعليم العالي الإسلامي وفقاً

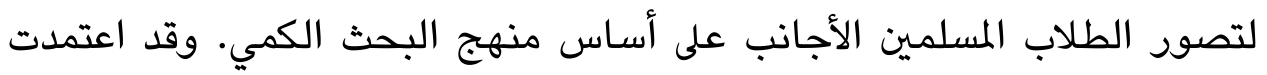

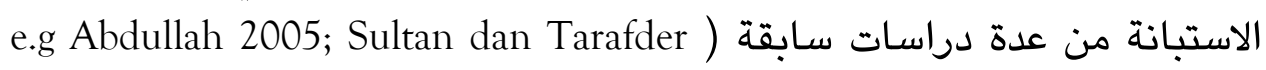
(2007; Annamdevula and Bellamkonda 2012 التي تتناسب مع ظروف التعليم العالي الإسلامي. وقد أجريت مناقشة مجموعة مركزة (FGD) كبداية لصياغ الأدوات مما يدعو خمسة طلاب بكالوريوس وخمسة طلاب الدراسات العليا الذين

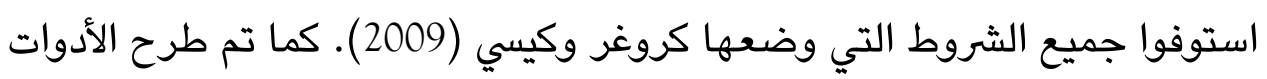


المصممة على الخبراء في مجال التعليم العالي الإسلامي وتسويق خدمات التعليم

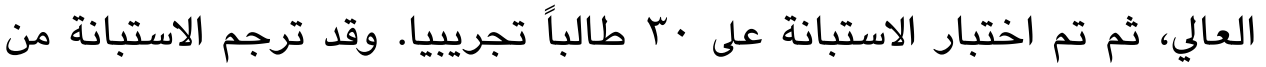
اللغة الإندونيسية إلى اللغة العربية (لطلاب الشرق الأوسط) واللغة الإنجليزية

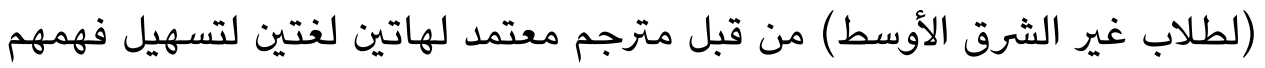

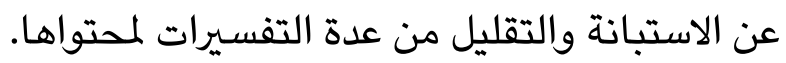
وقد أجري البحث في جامعةمولانا مالك إبراهيم الإسلامية الحكومية مالانج، لأنها طبقت نظام إدارة الجودة للمنظمة الدولية للمعايير (ISO) 9001:2015، وتمتلك الإنكا أكبر عدد للطلاب الأجانب بين الجامعات الإسلامية الأخرى في إندونيسيا بحيث إدارئ

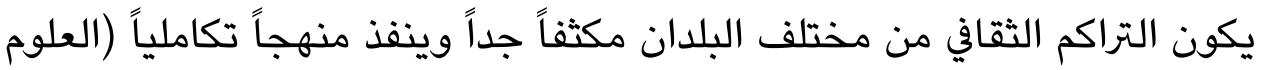
والإسلام). تم الحصول على بيانات البحث من خلال توزيع الاستبانات في نهاية عام 2019 (أكتوبر إلى ديسمبر). وأخذت 100 عينة تمثل خصائص السكان السكان

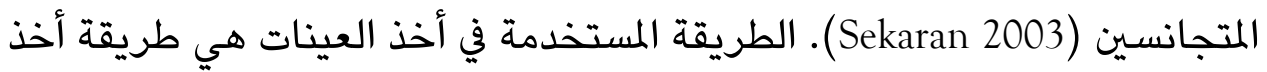
عينة عشوائية طبقية (أخذ العينة الاحتمالية) لأنها سهلة القيام بها، لديها مستوى كاف من الدقة ويمثل كل فئة فرعية من السكان نسبيا (Sekaran 2003) ـ استخدم مقياس ليكرت الخماسي (ا غير موافق بالشدة إلى ه موافق بالشدة) للاستبانة لأنه فئه

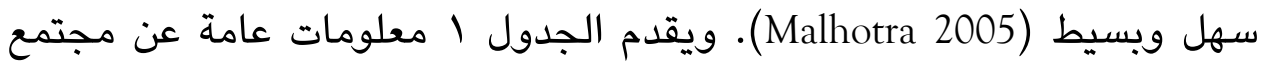

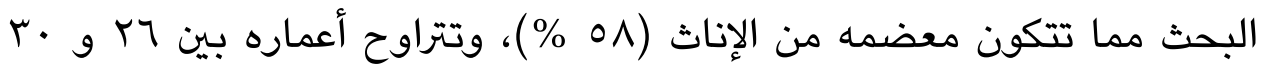

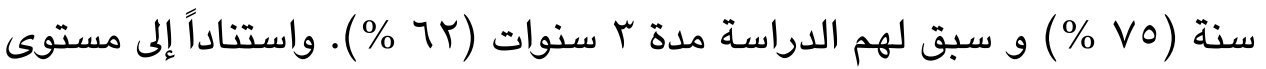
تعليمهم، فإن طلاب الدراسات العليا (ع^ \% \% ) ساهموا كثيرا وأكثرهم طلاب ليبيا . (\% r T )

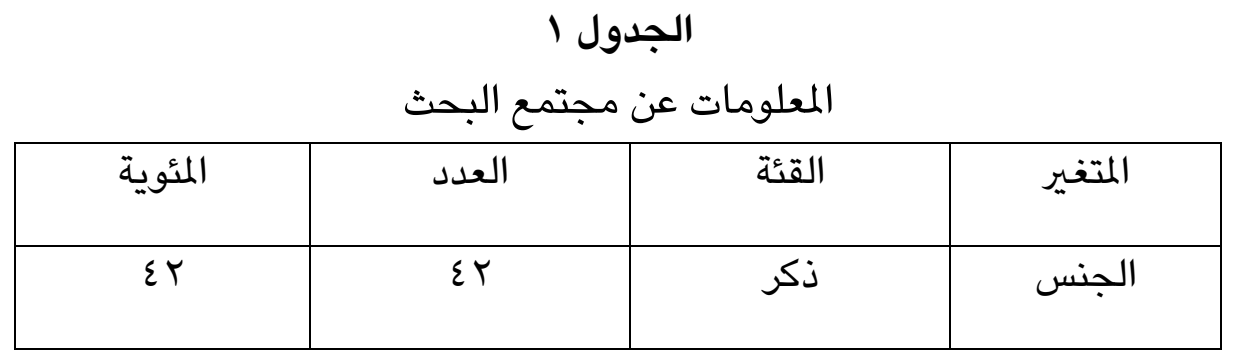




\begin{tabular}{|c|c|c|c|}
\hline $0 \wedge$ & $0 \wedge$ & أنثى & \\
\hline $1 \varepsilon$ & $1 \varepsilon$ & rO-r. & العمر \\
\hline Vo & Vo & $r \cdot-r\rceil$ & \\
\hline 11 & 11 & أكثر من · r & \\
\hline$\varepsilon$ & $\varepsilon$ & $r$ & مدة الدراسة \\
\hline $7 r$ & $7 r$ & $r$ & \\
\hline rY & YY & $\varepsilon$ & \\
\hline$\Lambda$ & $\Lambda$ & o & \\
\hline 17 & 17 & البكالوريوس & مستوى التعليم \\
\hline$\Lambda \varepsilon$ & $\wedge \varepsilon$ & الدراسـات العليا & \\
\hline 11 & 11 & ماليزيا & البلد \\
\hline 10 & 10 & تيلاند & \\
\hline$r$ & $r$ & الصين & \\
\hline 7 & 7 & السودان & \\
\hline 1 & 1 & مصر & \\
\hline rT & re & ليبيا & \\
\hline 0 & 0 & مداغسكر & \\
\hline$\varepsilon$ & $\varepsilon$ & روسيا & \\
\hline
\end{tabular}

Ulul Albab Volume 21, No.1 Tahun 2020 


\begin{tabular}{|c|c|c|c|}
\hline$\wedge$ & $\wedge$ & & \\
\hline$r$ & $r$ & كنغافور & \\
\hline$\varepsilon$ & $\varepsilon$ & صوماليا & \\
\hline$r$ & $r$ & تيمور الشرقية & \\
\hline
\end{tabular}

المصدر : حساب برنامج SPSS

النتائج والمناقشـات

أظهرت خلاصة النتائج في الجدول r مقياس كايزر - ماير - أولكين (KMO)

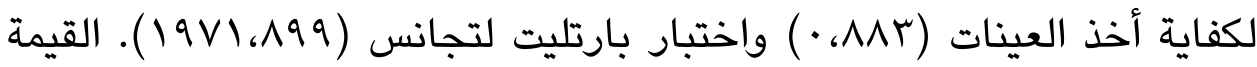
أكثر من 0، · ويتم التصريح عن تحقيق العينة الكافية (Malhotra et al. 2017). اختبار بارتليت لتجانس قيمته كبير (أقل من ه •، • ) لذلك يمكن القيام بتحليل

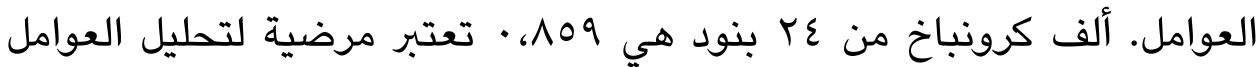

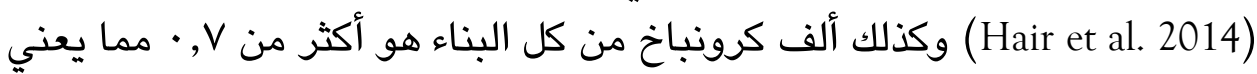

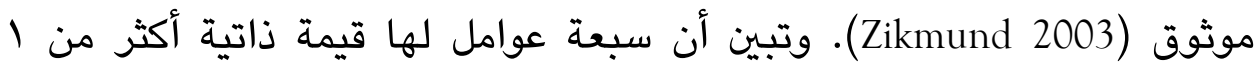

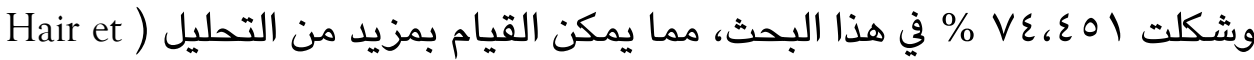
(al. 2014; Malhotra et al. 2017 من ع ع بنداً تم تجميعها في خمسة عوامل وحققت التحميل الموصى به لأكثر من بr، · مما يمثل أعلى عامل تحميل (Malhotra et al. 2017). ونتيجة لذلك، فإن الأبعاد المتعلقة بجودة الخدمات المتصورة في مؤسسات التعليم

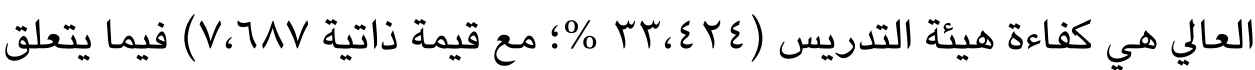

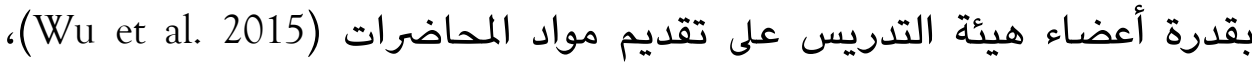

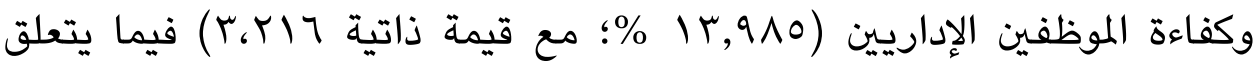
بخدمات الموظفين الداعمين في مؤسسات التعليم العالي الإسلامي كما توصل إليه

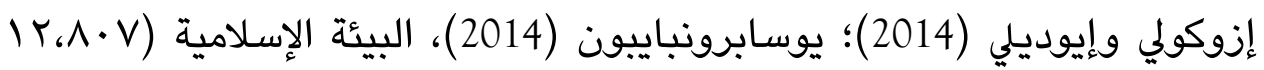

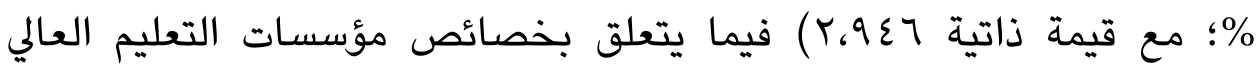




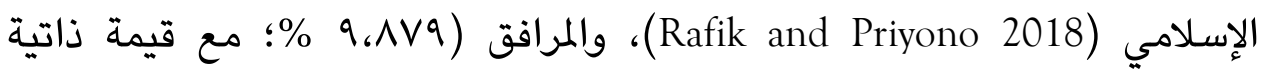

Owlia and ( مما يعتبر بتقويم الطلاب على الخدمات في التعليم العالي (Y.TVY Aspinwall 1996; Hanaysha et al. 2011; Singh and Kumar 2014; (Teeroovengadum et al. 2016 قيمة ذاتية r ..،1) وهي أيضاً من أبعاد هامة في مؤسسات التعليم العالي .(Lagrosen et al. 2004; Teeroovengadum et al. 2016)

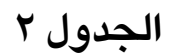

نتائج تحليل العوامل في التصور عن جودة الخدمات في مؤسسة التعليم العالي الإسلامية

\begin{tabular}{|c|c|c|c|c|c|}
\hline \multicolumn{5}{|c|}{ 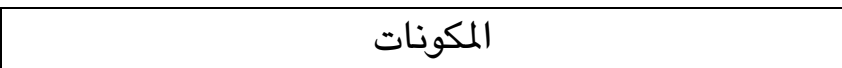 } & \multirow[t]{2}{*}{ بنود البحث } \\
\hline التكنولوجي & 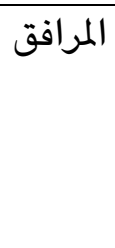 & الإسلاه & الموظفين & كفاءة هيئة & \\
\hline & & & $\Lambda \vee 1$. & & جيدواصل الموظف الإداري بشكل \\
\hline & & & $\Lambda \cdot \varepsilon$. & & يخدم الموظف الإداري بالإخلاص \\
\hline & & & $\wedge \vee\urcorner$. & & يخدم الموظف الإداري بالودية \\
\hline & & & q.r. & & يتصرف الموظف الإداري بأدب \\
\hline & & & V^T. & & يحترم الموظف الإداري الطلاب \\
\hline & & & $\Lambda \vee r$. & & تسهل مقابلة الموظف الإداري \\
\hline $9 \wedge r$. & & & & & تلجامر خدمات الإنترنت في الحرم \\
\hline
\end{tabular}




\begin{tabular}{|c|c|c|c|}
\hline 9.1. & & & 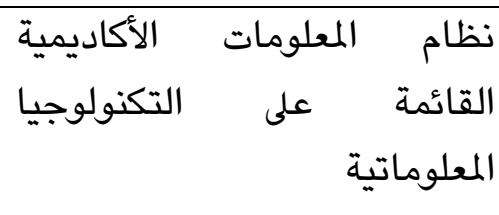 \\
\hline & $\Lambda \cdot 9$. & & الميزات المعمارية لمبنى الحرم \\
\hline & $\wedge \varepsilon \varepsilon$. & & المسجد \\
\hline & $\wedge \backslash \wedge$. & & سكن الطعاليم الإسلامية الذي تعطي فيه \\
\hline & MIr. & & 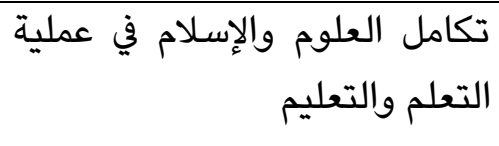 \\
\hline & $\Lambda \vee \cdot$. & & المنهج الدراسي في التكامل بين \\
\hline & & $\vee \wedge \cdot$. & تكنولوجيا معلوماتية في التعليم \\
\hline & & VAr. & تقدم هيئة التدريس معلومات عن \\
\hline & & $\vee \wedge \cdot$. & بأدب تتعامل هيئة التدريس طلابهم \\
\hline & & VIV. & تمتلك هيئة التدريس الكفاءة \\
\hline & & 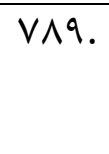 & نتقن هيئة التدريس معرفة \\
\hline
\end{tabular}




\begin{tabular}{|c|c|c|c|c|c|}
\hline & $\Lambda \vee r$. & & & & مواقف السيارات \\
\hline & $\wedge 11$. & & & & سكن الطلاب \\
\hline & Arr. & & & & الصالة الرياضية \\
\hline & Aro. & & & & خدمات المكتبة إلكتورنيا \\
\hline & & & & ヘтr. & تحفز هيئة التدريس طلابهم \\
\hline & & & & $9 \cdot \Lambda$. & تستعد هيئة التدريس في مساعدة \\
\hline $1, \cdots r$ & T,YVT & $r, 9 \varepsilon 7$ & r,YIT & $\vee,\urcorner \wedge \vee$ & القيم الذاتية الأولية \\
\hline$\varepsilon, r \circ V$ & $q, \wedge \vee q$ & 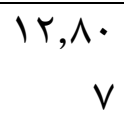 & $1 r, 9 \wedge 0$ & $r \mu, \varepsilon Y \varepsilon$ & النسبة المئوية للتجانس \\
\hline$V \varepsilon, \varepsilon 01$ & $\begin{array}{r}V \cdot, \cdot q \\
\varepsilon\end{array}$ & $\begin{array}{r}7 \cdot, Y 1 \\
0\end{array}$ & $\varepsilon \vee, \varepsilon \cdot \Lambda$ & $r \mu, \varepsilon Y \varepsilon$ & النسبة التراكمية \\
\hline \multirow[t]{6}{*}{$\cdot, \wedge \cdot r$} & $\cdot, \wedge 00$ & $\cdot, 97$. & $\cdot, \wedge 97$ & $\cdot 9 \mathrm{TV}$ & ألفا كرونباخ \\
\hline & & & & $V \varepsilon, \varepsilon \circ 1$ & مجموع النسبة المئوية للتباين \\
\hline & & & & $\cdot, 109$ & كرونبانخ (عدد عناصر : ع r) \\
\hline & & & & $\cdot, \wedge \wedge \uparrow$ & 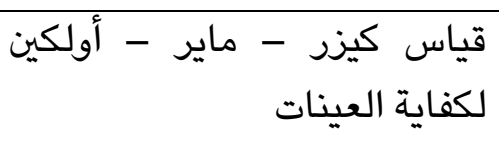 \\
\hline & & & & $\begin{array}{r}19 \vee 1,19 \\
9\end{array}$ & 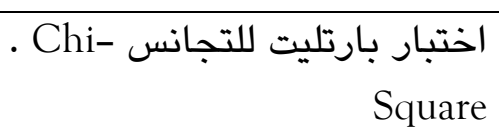 \\
\hline & & & & ror & $\mathrm{df}$ \\
\hline
\end{tabular}




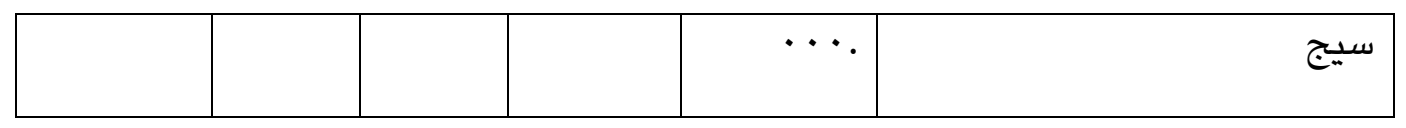

المصدر: حساب برنامج SPSS

\section{مناقشة نتائج البحث}

الهدف من هذا البحث هو إيجاد الأبعاد من تصور جودة الخدمات في مؤسسة التعليم العالي الإسلامية، وهو تجرية الطلاب المسلمين الأجانب الذين هودين درسوا في هذه الجامعة. واستند البحث إلى الأدوات التي اعتمدت من بحوث الجهابه سابقة

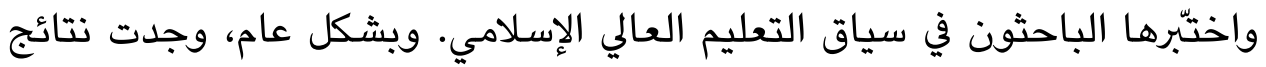
هذا البحث أن التصور عن جودة الخدمات الجيدة في نظرة الطلاب المسلمين الأجانب الذين درسوا في التعليم العالي الإسلامي (جامعة مولانا مالك إبراهيم الإسلامية الحكومية مالانج) تشمل أولا: كفاءة هيئة التدريس كما توصلت إليه أوليا وأسفين وول (1996)؛ لاغروسين وآخرون (2004)؛ سريكتانيو وغنوث (2005)؛ عبد الله (2006)؛ سينتيل كومار وأرولراج (2011)؛ أناميديفولا وبيلاميكوندا (2012)؛ تيروفينفادوم وآخرون (2016). ثانياً: كفاءة الموظفين الإداريين التي تشـير إلى قدرة الموظفين التقنيين على مساعدة الطلاب على خدمة

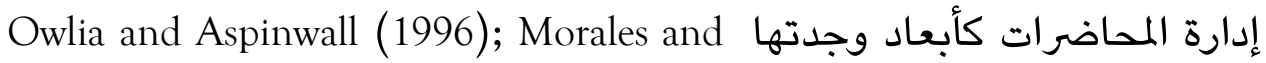
Calderón (1999); Srikatanyoo and Gnoth (2005); Abdullah (2006); Ling et al (2010); Annamdevula and Bellamkonda (2012); Ereş and Clothey

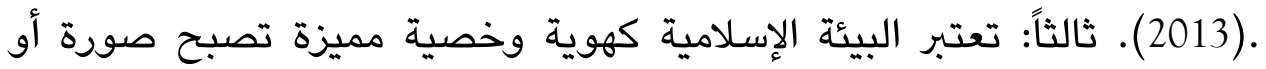
علامة تجارية تمايز بين مؤسسة التعليم العالي الإسلامية والعلمانية. هذه الحقيقة والحسيه

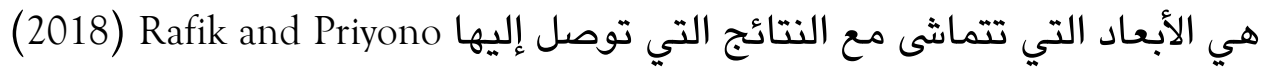

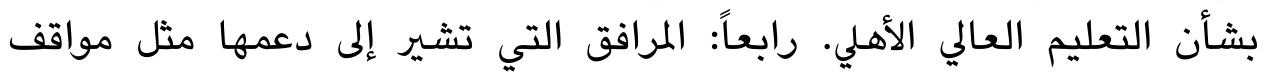

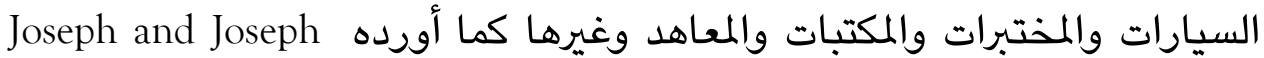
(1997); Morales and Calderón (1999); Lagrosen et al (2004); Hanaysha et al (2011); Annamdevula and Bellamkonda (2012); Teeroovengadum et al (2016). وأخيراً: وجود خدمة التكنولوجيا المعلوماتية التي تحدد جودة الخدمات 
Lagrosen et al في التعليم العالي بما يتماشى مع نتائج الدراسات التي أجرها (2004); Ling et al (2010). ساعدت هذه الأدوات كلها في تحقيق الثقافة الإسلامية في جامعة مولانا مالك إبراهيم الإسلامية الحكومية مالانج. ويرد في الجدول r ملخص الدراسات السابقة المتصل بنتائج البحث. موند

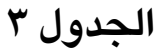

أبعاد التصور عن جودة الخدمات في مؤسسة التعليم العالي الإسلامية - تجرية الطلاب

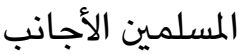

\begin{tabular}{|rr|rr|r|}
\hline & & \\
\hline \\
\hline
\end{tabular}




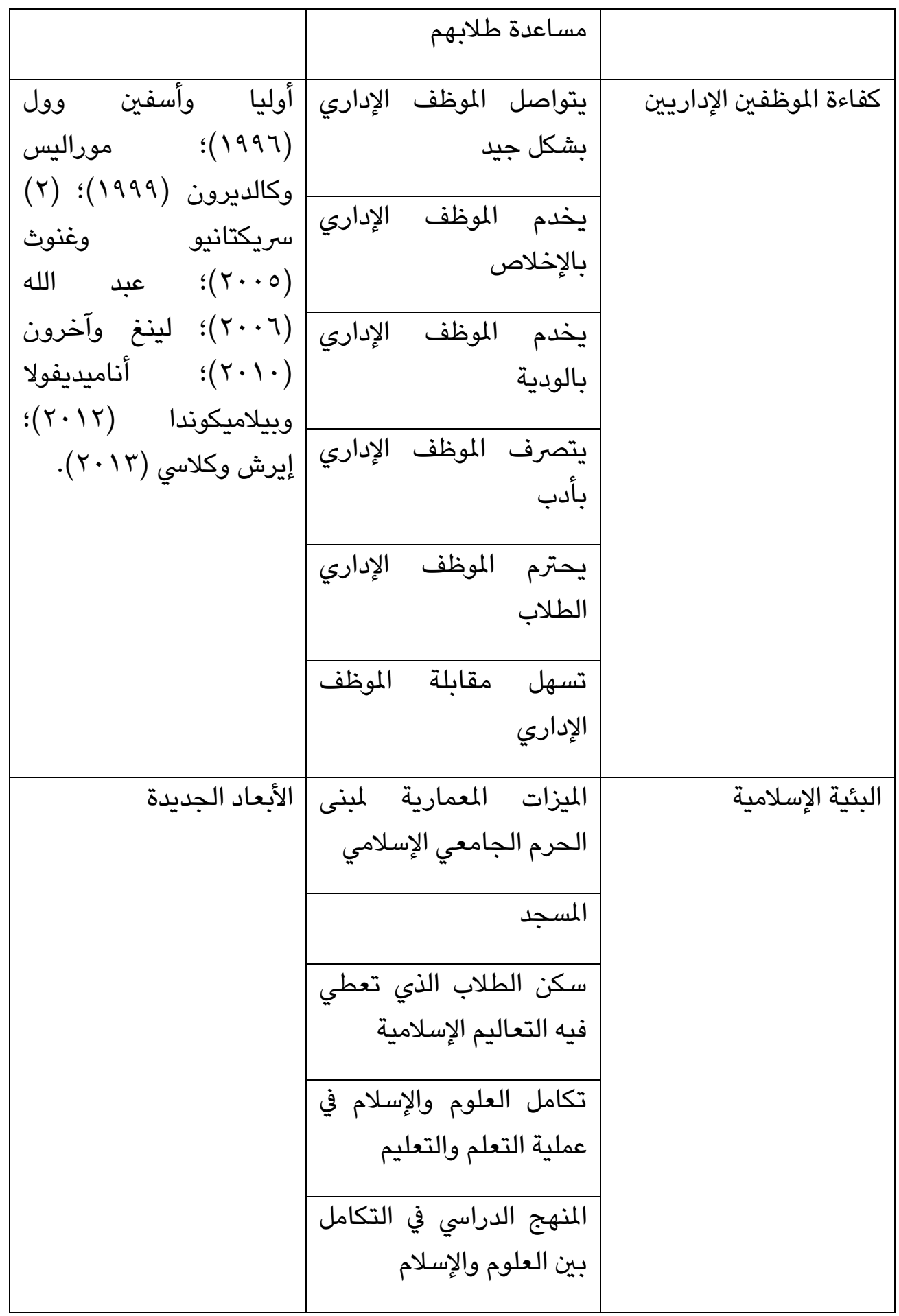

Ulul Albab Volume 21, No.1 Tahun 2020 


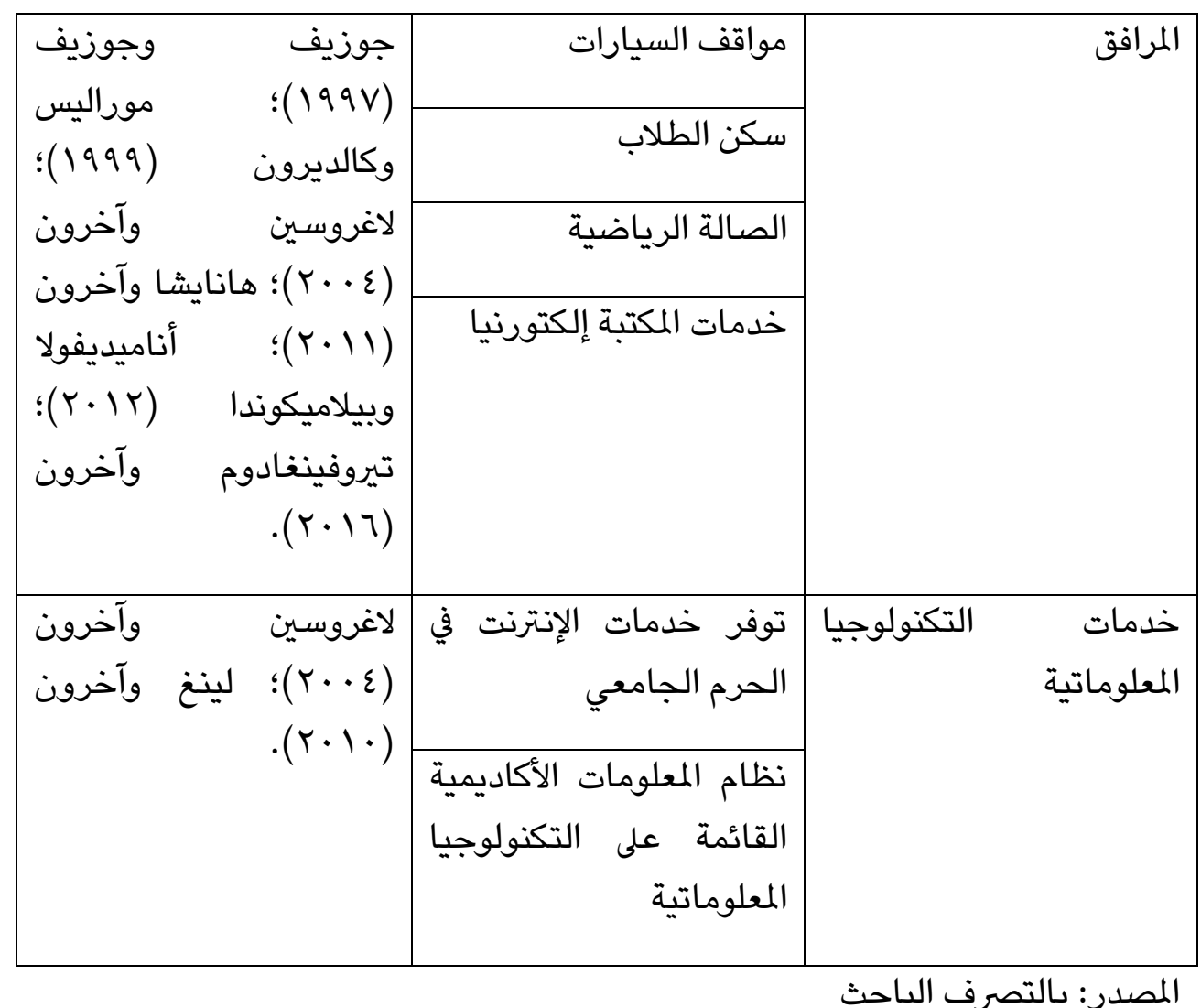

الآثار المترتبة من البحث

تجري البحوث المتعلقة بتصور جودة الخدمات في التعليم العالي في الجامعات الحكومية عموما (Sumaedi et al. 2012) والأهلية ( Rafik and Priyono 2018;

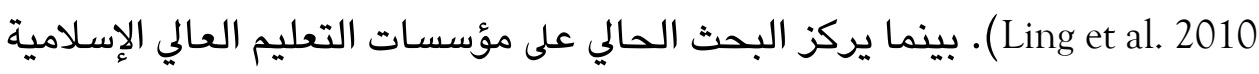

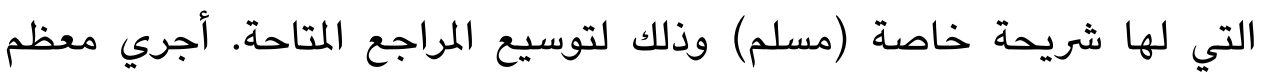

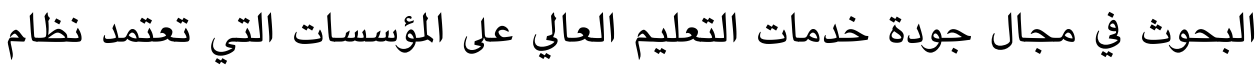

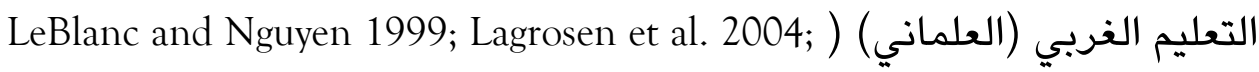
(Teeroovengadum et al. 2016 وسعى هذا البحث إلى إيجاد أبعاد خاصة في سياق مؤسسات التعليم العالي الإسلامية في إندونيسيا استناداً إلى تجرية الطلاب المسلمين الأجانب الذين يتميزون بهذا البحث. 
ريما بناء التصور عن جودة الخدمات بالنسبة للمديرين في مؤسسة التعليم العالي الإسلامية التي تطبق معيار ISO (كما في جامعة مولانا مالك إبراهيم الإسلامية

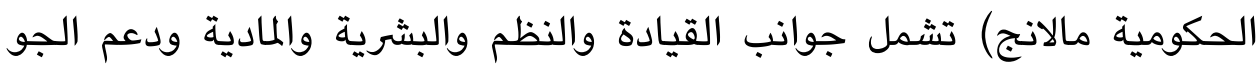

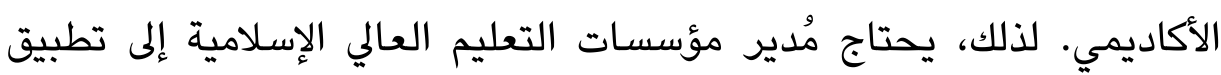

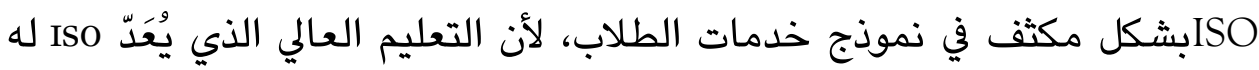
جودة مختلفة عن التعليم العالي الذي لا يطبقه (Sakthivel et al. 2005). بالإضافة لإنة إلى ذلك، يجب على مدير مؤسسة التعليم العالي الإسلامية الاهتمام بأبعاد كفاءة

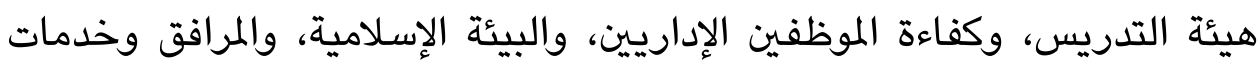

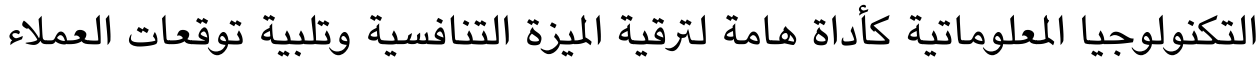
(Abdullah 2005)

وللباحثين، لا يمكن استخدام عينات البحث التي تستهدف الطلاب المسلمين الأجانب لتعميم الطلاب المسلمين في أنحاء إندونيسيا في سياق التعليم العات العالي الإسلامي بإندونيسيا، ويالتالي تحتاج إلى دراسة أوسع لتحقيق نتائج أكثر ملاءمة.

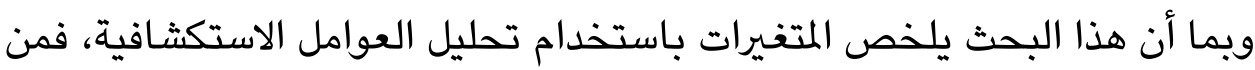

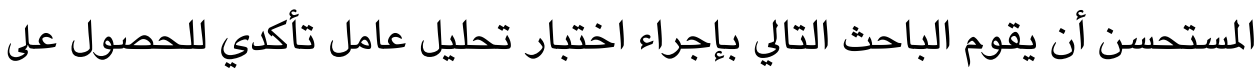

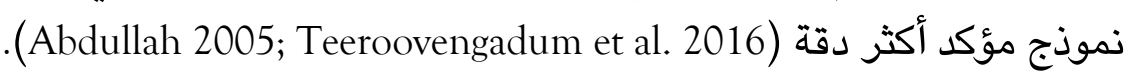

\section{References}

Abdullah, Firdaus. 2005. "HEdPERF Versus SERVPERF The Quest For Ideal Measuring Instrument of Service Quality in Higher Education Sector." Quality Assurance in Education 13(4): 305-328.

Abdullah, Firdaus. 2006. "The Development of Hedperf: A New Measuring Instrument of Service Quality for the Higher Education Sector." International Journal of Consumer Studies 30(6): 569-581.

Abidin, Munirul. 2015. “Students' Perception of Service Quality Dimensions in Islamic Higher Education." International Journal of Innovative Research $\mathcal{E}$ Development 4(1): 237-242.

Aga, Mehmet and Okan Safakli. 2007. "An Empirical Investigation of Service Quality and Customer Satisfaction in Professional 
Accounting Firms: Evidence from North Cyprus." Problems and Perspectives in Management 5(3): 84-98.

Alavi, Hamid Reza. 2007. "Al-Ghazali on Moral Education." Journal of Moral Education 36(3): 309-319.

Annamdevula, Subrahmanyam and Raja Shekhar Bellamkonda. 2012.

"Development of HiEdQUAL for Measuring Service Quality in Indian Higher Education Sector." International Journal of Innovation, Management and Technology 3(4): 412-416.

Arokiasamy, Anantha Raj A. and Abdul Ghani Abdullah. 2012. "Service Quality and Students Satisfaction at Higher Learning Institutions: A Case Study of Malaysian University Competitiveness." International Journal of Management and Strategy 3(5): 1-16.

Asaduzzaman, Mohammad, Moyazzem Hossain, and M. Habeebur Rahman. 2013. "Service Quality and Student Satisfaction: A Case Study on Private Universities in Bangladesh.” International Journal of Economics, Finance and Management Sciences 1(3): 128-135.

Brown, Robert M. and Timothy William Mazzarol. 2009. "The Importance of Institutional Image to Student Satisfaction and Loyalty Within Higher Education." High Educ 58: 81-95.

Calvo-Porral, Cristina, Jean-Pierre Lévy Mangin and M. Isabel Novo-Corti. 2013. "Perceived Quality in Higher Education: An Empirical Study." Marketing Intelligence Eु Planning 31(6): 601-619.

Chen, Chun-Fu and Chin-Tsu Chen. 2014. "The Effect of Higher Education Brand Images on Satisfaction and Lifetime Value from Students Viewpoint.” Anthropologist 17(1): 137-145.

Clemes, Michael D., Christopher Gan and Min Ren. 2008. "An Empirical Analysis of Customer Satisfaction in International Air Travel." Innovative Marketing 4(2): 50-62.

Dado, Jaroslav, Janka Tabotrecka Petrovicova, Sreten Cuzovic and Tamara Rajic. 2012. "An Empirical Examination of The Relationships Between Service Quality, Satisfaction and Behavioral Intentions in Higher Education Setting." Serbian Journal of Management 7(2): 203 . 218. 
Direktorat Pendidikan Tinggi Islam (DIKTIS). 2019. "Perguruan Tinggi Keagamaan Islam (Islamic Religious College).” Direktorat Jenderal Pendidikan Islam Kementerian Agama Republik Indonesia, available at: http://diktis.kemenag.go.id/bansos/cari_nspt.php (accessed July 11, 2019).

Dunworth, Katie, Trevor Grimshaw, Janina Iwaniec, \& James McKinley. 2019. "Language and The Development of Intercultural Competence in an 'Internationalised' University: Staff and Student Perspectives." Teaching in Higher Education, 1-16.

Ekinci, Yuksel, Popi Prokopaki, and Cihan Cobanoglu. 2003. "Service Quality in Cretan Accommodations: Marketing Strategies for the UK Holiday Market." International Journal of Hospitality Management 22(1): 47-66.

Ereş, Figen and Rebecca Clothey. 2013. "Perceived Service Quality in Schools of Education: A Comparative Study between Drexel University and Gazi University." American International Journal of Social Science 2 (7): 6-15.

Ezeokoli, Rita Nkiruka and Ayodele Kalowale O. 2014. "Dimensions of Service Quality Encountered by Students on Sustainability of Higher Education in Nigeria." Developing Country Studies 4(6): 147156.

Furco, Andrew, \& Kris Lockhart. 2018. "Building a University-Wide Agenda for Intercultural Competence and Understanding: Lessons Learned at the University of Minnesota.” Metropolitan Universities 29(1): 77-92.

Furer, O.livier, Ben Shaw-Ching Liu and Devanathan Sudharshan. 2000. "The Relationships between Culture and Service Quality Perceptions -Basis for Cross- Cultural Market Segmentation and Resource Allocation." Journal of Service Research 2(4): 355-371.

Gruber, Thorsten, Stefan Fuss Roediger Voss and Michaela Glaeser-Zikuda. 2010. "Examining Student Satisfaction With Higher Education Services: Using A New Measurement Tool." International Journal of Public Sector Management 23(2): 105-123. 
Hair, Joseph F., William C. Black, Barry J. Babin and Rolph E. Anderson. 2014. Multivariate Data Analysis, Seventh Edition, Pearson Education Limited, Edinburgh Gate Harlow Essex England, London.

Hanaysha, Jalal R. M., Halim Hilman Abdullah, and Ari Warokka. 2011. "Service Quality and Students Satisfaction at Higher Learning Institutions: The Competing Dimensions of Malaysian Universities Competitiveness." Journal of Southeast Asian Research 2011: 1-10.

Hill, Yvonne, Laurie Lomas and Janet MacGregor. 2003. "Students' Perceptions of Quality in Higher Education." Quality Assurance in Education 11(1): 15-20.

Hofstede, Geert. 2011. "Dimensionalizing Cultures: The Hofstede Model in Context." Online Readings in Psychology and Culture 2(1): 1-26.

Holdford, David and Thomas P. Reinders. 2001. "Development of an Instrument to Assess Student Perceptions of the Quality of Pharmaceutical Education." American Journal of Pharmaceutical Education 65(2): 125-131.

Hussain, Khuram. 2007. "An Islamic Consideration of Western Moral Education: An Exploration of the Individual." Journal of Moral Education 36(3): 297-308.

Hutapea, Bonar. 2014. "Stres Kehidupan, Religuisitas, dan Penyesuaian Diri Warga Indonesia sebagai Mahasiswa Internasional.” Jurnal Makara Hubs-Asia 18(1): 25-40.

Ivy, Jonathan. 2001. "Higher Education Institution Image: Acorrespondence Analysis Approach." International Journal of Educational Management 15(6): 276-282.

Joseph, Mathew and Beatriz Joseph. 1997. "Service Quality in Education: A Student Perspective." Quality Assurance in Education 5(1): 15-21.

Kelso, Richard Scott. 2008. Measuring undergraduate student perceptions of service quality in higher education. Graduate theses and dissertations. Department of Adult, Career, and Higher Education College of Education University of South Florida. 
Krueger, Richard. A. and Mary Anne Casey. 2000. Focus Groups: A Practical Guide for Applied Research: SAGE.

Lagrosen, Stefan, Roxana Seyyed-Hashemi and Marcus Leitner. 2004. "Examination of the Dimensions of Quality in Higher Education." Quality Assurance in Education 12(2): 61-69.

LeBlanc, Gaston and Nha Nguyen. 1999. "Listening to the Customer's Voice: Examining Perceived Service Value among Business College Students." International Journal of Educational Management 13(4): 187 198.

Ling, Kwek Choon, Lau Teck Chai and Hoi Piew Tan. 2010. "The 'InsideOut' and 'Outside-In' Approaches on Students Perceived Service Quality: An Empirical Evaluation." Management Science and Engineering 4(2): 1-26.

Malhotra, Naresh K. 2005. Riset Pemasaran Pendekatan Terapan Edisi Keempat Jilid 1 dan 2. Alih Bahasa Soleh Rusyadi Maryam. Jakarta: PT. Indeks Kelompok Gramedia.

Malhotra, Naresh K., Daniel Nunan and David F. Birks. 2007. Marketing Research: An Applied Approach, Fifth Edition, Pearson Education Limited Edinburgh Gate Harlow United Kingdom.

McKinley, Jim, Katie Dunworth, Trevor Grimshaw \& Janina Iwaniec. 2019. "Developing Intercultural Competence in A 'Comfortable' third Space: Postgraduate Studies In The UK." Language and Intercultural Communication 19(1): 9-22.

Melewar, T. C. and Sibel Akel. 2005. "The role of corporate identity in the higher education sector A case study." Corporate Communications: An International Journal 10(1): 41-57.

Mitasari, Zuni \& Yuswa Istikomayanti. 2018. Hubungan Antara Culture Shock dengan Hasil Belajar Mahasiswa Tahun Pertama. Jurnal Psikologi Pendidikan dan Konseling 4(2): 105-113.

Morales, Miguel and Luis F. Calderón. 1999. "Assessing Service Quality in Schools of Business: Dimensions of Service Quality in Continuing Professional Education (CPE)." Journal of Economics, Finance and Administrative Science 5(9-10): 125-141. 
Omar, Nor Asiah, Muhammad Azrin Nazri, Nur Khalidah Abu, and Zoharah Omar. 2009. "Parents Perceived Service Quality, Satisfaction and Trust of A Childcare Centre: Implication On Loyalty." International Review of Business Research Papers 5(5): 299-314.

Owlia, Mohammad S. and Elaine M. Aspinwall. 1996. "A Framework for the Dimensions of Quality in Higher Education." Quality Assurance in Education 4(2): 12-20.

Parasuraman, A. Parsu, Valarie A. Zeithaml, and Leonard L. Berry. 1985. "A Conceptual Model of Service Quality and Its Implications for Future Research.” Journal of Marketing 49: 41-50.

Pyvis, David, \& Anne Chapman. 2005. "Culture Shock and The International Student 'Offshore'." Journal of research in international education 4(1): 23-42.

Pinar, Musa, Sandra E. Strasser, L. Schiffel, and J. M. Stück. 2014. "Effects of Gender Similarity/Dissimilarity, Gender Stereotyping and Culture On." Journal of Organizational Culture, Communications and Conflict 18(1): 149-169.

Rafik, Abdur and Anjar Priyono. 2018. "A New Insight into Alumni Satisfaction Model for Islamic Higher Education Institutions (IHEI)." Management Research Review 41(12): 1411-1437.

Rasli, Aamran, Ahmadreza Shekarchizadeh, and Muhammad Jawad Iqbal. 2012. "Perception of Service Quality in Higher Education: Perspective of Iranian Students in Malaysian Universities." International Journal of Academic Research in Management (IJARM) 1(1): 10-25.

Rifai, Nurlela, Fauzan Fauzan, Wahdi Sayuti, and Bahrissalim. 2014. "Integrasi Keilmuan dalam Pengembangan Kurikulum di UIN SeIndonesia: Evaluasi Penerapan Integrasi Keilmuan UIN dalam Kurikulum dan Proses Pembelajaran (Scientific Integration in Curriculum Development at UIN throughout Indonesia: Evaluation of The Application of UIN Scientific Integration in The Curriculum and Learning Process)." Tarbiya 1(1): 13-34.

Saglik, Erkan, Ali Caglar Gulluce, U. Kaya, and Caglar Kadir Ozhan. 2014. "Service Quality and Customer Satisfaction Relationship: A 
Research in Erzurum Ataturk University Refectory." American International Journal of Contemporary Research 4(1): 100-117.

Sakthivel, P. B., G. Rajendran, and Ramasamy Raju. 2005. "TQM Implementation and Students' Satisfaction of Academic Performance." The TQM Magazine 17(6): 573-589.

Sekaran, Uma, \& Roger Bougie. 2016. Research Methods for Business: A Skill Building Approach, John Wiley and Sons Inc, New York, NY.

Senthilumar, Nakkeeran, and A. Arulraj. 2011. "SQM-HEI Determination of Service Quality Measurement of Higher Education in India." Journal of Modelling in Management 6 (1): 60-78.

Singh, Gurbinder and Maneek Kumar. 2014. "Exploratory Factor Analysis of Service Quality Dimensions for Higher Educational Institutes: A Students Perspective." Global Journal of Management and Business Research: An Administration and Management 14(8): 38-48.

Srikatanyoo, Natthawut and Jurgen Gnoth. 2005. "Quality Dimensions in International Tertiary Education: A Thai Prospective Students Perspective." Quality Management Journal 12(1): 30-40.

Sultan, Parves and Tasmiha Tarafder. 2007. "A Model for Quality Assessment in Higher Education: Implications for ODL Universities." Malaysian Journal of Distance Education 9(2): 125-143.

Sumaedi, Sik, Gede Bakti, and Nur Metasari. 2012. "An Empirical Study of State University Students Perceived Service Quality." Quality Assurance in Education 20(2) 164-183.

Teeroovengadum, Viraiyan, T. J. Kamalanabhan, and Ashley Keshwar Seebaluck. 2016. "Measuring Service Quality in Higher Education: Development of a Hierarchical Model (HESQUAL)." Quality Assurance in Education 24(2): 244-258.

Telford, Ronnie and Ron Masson. 2005. "The Congruence of Quality Values in Higher Education." Quality Assurance in Education 13(2): 107-119.

Wu, Yu-Chi, Chin-Shih Tsai, Hsiao-Wen Hsiung, and Kuan-Ying Chen. 2015. "Linkage between Frontline Employee Service Competence 
Scale and Customer Perceptions of Service Quality." Journal of Services Marketing 29(3) 224-234.

Yasin, Raudlotul Firdaus Fatah and Mohd. Shah Jani. 2013. "Islamic Education: The Philosophy, Aim, and Main Features." International Journal of Education and Research 1(10): 1-18.

Yousapronpaiboon, Khanchitpol. 2014. "SERVQUAL: Measuring Higher Education Service Quality in Thailand." Procedia - Social and Behavioral Sciences 116: 1088-1095.

Zenner, Keni \& Dian Squire. 2020. "International Student Success: Exploring the Intercultural Competence of Academic Advisors". Journal of Student Affairs Research and Practice: 1-14.

Zikmund, William G., Babin, B. J., Carr, J. C., \& Griffin, M. 2003. Business Research Methods, $\left(7^{\text {th }}\right.$ ed.). Thomson/South-Western. 